\title{
Interaction Design for a Pet-Like Remote Control
}

\author{
Kazuki Kobayashi $^{1}$, Yutaro Nakagawa ${ }^{2}$, Seiji Yamada ${ }^{3,4}$, \\ Shinobu Nakagawa ${ }^{2}$, and Yasunori Saito ${ }^{5}$ \\ 1 Graduate School of Science and Technology, Shinshu University \\ 4-17-1 Wakasato, Nagano City, 380-8553 Japan \\ kby@shinshu-u.ac.jp \\ 2 Design Department,Osaka University of Arts \\ 469 Higashiyama, Kanan-cho, Minami Kawachi-gun Osaka, 585-8555 Japan \\ shinobu@osaka-geidai.ac.jp, yutarou19870307@yahoo.co.jp \\ 3 National Institute of Informatics \\ 2-1-2 Hitotsubashi, Chiyoda, Tokyo, 101-8430 Japan \\ seiji@nii.ac.jp \\ 4 SOKENDAI \\ Shonan Village, Hayama, Kanagawa, 240-0193 Japan \\ 5 Faculty of Engineering, Shinshu University \\ 4-17-1 Wakasato, Nagano City, 380-8553 Japan \\ saitoh@cs.shinshu-u.ac.jp
}

\begin{abstract}
This paper describes a novel remote control operable with stroking its surface. Advantages of the developed remote control are high familiarity and stroke operation. Those enable users to have familiarity with it and to use it without looking at the fingers. We apply it to an interaction system with TV. The proposed system has the tolerance for mistakes in comparison with conventional button-based remote controls because it enables unfamiliar users to home electric appliances to use it casually without fear of mistakes and unexpected behavior.
\end{abstract}

Keywords: remote control agent, pet-like embodied agent, partial execution.

\section{Introduction}

Various remote controls are used in our home. Home electronics such as televisions, air conditioners, room lights usually have remote controls. They are currently commonplace devices. However, many remote controls confuse people when they use a home electric appliance. Universal remote 1 that aggregates functions of various remote controls is one of technical solutions for this problem. On the other hand, users need to search a button that they want to operate and to correctly move the fingers to push it. It is not highly problematic for young people, but elderly people have a difficulty in operating remote controls. It will be comfortable to use a remote control without looking at their hands. 

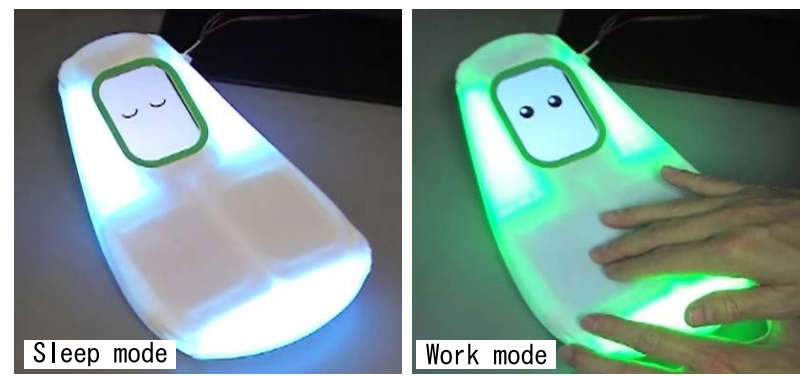

Fig. 1. Remote Control Agent: Rebo

Such a remote control is also comfortable to young people familiar to home electric appliances.

In this paper, we propose a remote control, Rebo, like a pet animal. Users stroke its surface with the fingers to control various home electric appliances. Advantages of Rebo are high familiarity and stroke operation. Its life-like appearance and facial expressions make it familiar with users. They can easily use it without looking at the fingers by stroking. Therefore, users do not have to seek a button that they want to operate and use it smoothly.

We develop an interaction system with TV as an example of the proposed remote control. Rebo enables users to easily execute functions of the TV.

\section{Pet-Like Remote Control Agent}

Figure 1] shows the developed pet-like remote control agent, Rebo. Rebo is an agent of remote controls of home electric appliances. It has a user-friendly appearance; a smooth surface for stroking and a back side fit for users' thighs. It is $249 \mathrm{~mm}$ long, $146 \mathrm{~mm}$ wide, and $96 \mathrm{~mm}$ high. A small LCD monitor, full-color LED modules, and three touch sensors are embedded in the body. The touch sensors are covered with a soft and smooth cloth.

\subsection{Familiarity}

Rebo has a life-like appearance and facial expressions to acquire familiarity with a user (Fig. 1). We adopt the concept of "intermediate entity between artifacts and animate beings". Rebo is not only a tool but also a partner like a pet animal. The body of Rebo is covered with soft and bouncy cloth and it is pleasant to the touch. When the user strokes Rebo, it changes its facial expressions to inform him or her of various emotional states of Rebo. We consider this makes the user more comfortable with Rebo. Therefore, the user can enjoy interacting with Rebo. We believe that this concept plays an important role for establishing familiarity between users and Rebo. 


\subsection{Stroke Operation}

Rebo has no button and a user strokes its surface to control electric appliances. In this kind of operation, he or she does not need to move the fingers correctly and not seek the button that he or she wants. It is more comfortable for the user to gaze at an appliance than to gaze at the remote control, because the feedback from the appliance is more important than that from the remote control. However, the operation by stroking is different from the operation by pushing a button in a physical feeling. We then use LED lighting as an acknowledgment of the operation.

The advantage of the LED lighting has less interference against auditory and visual information. If an electric appliance such as a TV provides auditory information, it is inadequate that a remote control provides auditory feedback. In contrast, expressions by simple LED lighting can be grasped by users from their peripheral vision. We call this method "Peripheral feedback". Peripheral feedback has less interference against their central visual field. This method is similar to peripheral display [23], and its concept is based on our view that "the central player is the appliance and the backseat player is the remote control".

\section{TV Remote Agent}

We developed a TV remote agent based on the proposed remote control agent to implement all of the advantages in the previous section. In addition, we also designed feedback behavior of the TV and constructed an interaction system.

\subsection{Stroke Operation}

Functions we implemented in the system are channel switch, sound volume change, and power on/off. The TV screen was implemented on a PC. In this section, operation methods of Rebo and feedback behavior of the TV are described.

Channel Switch. Figure 2 shows a series of screenshots in which the positions of two video pictures are changed as a user strokes the surface of Rebo right and left. When the user widely strokes the surface of Rebo with her fingers, a video picture goes the outside of the TV frame and another video picture comes into the frame. When the user strokes Rebo for a short time, a part of another video
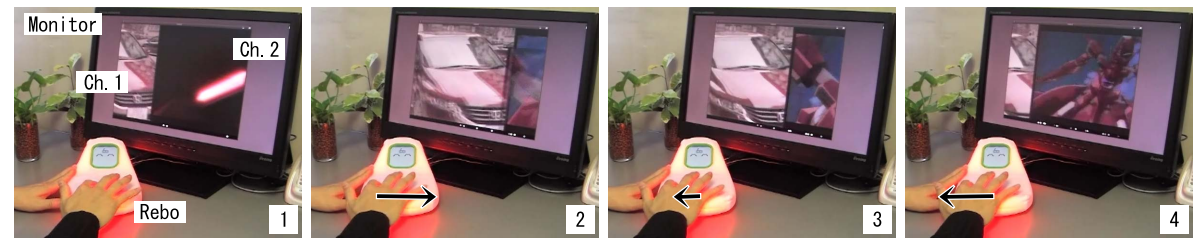

Fig. 2. Channel Switching 

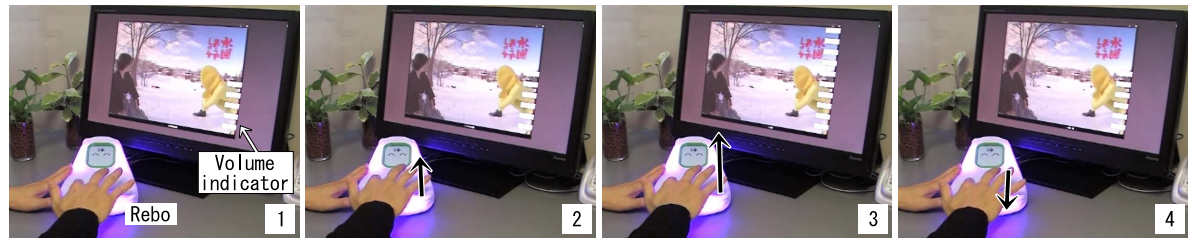

Fig. 3. Volume of Sound Changing
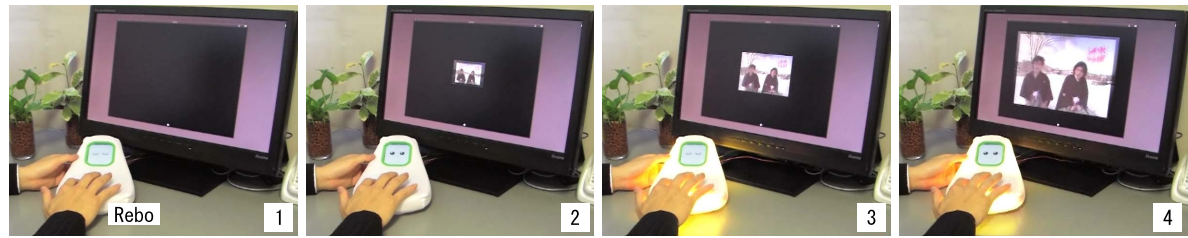

Fig. 4. Turn On

picture comes into the frame and then goes out of the frame automatically. In this implementation, we used recorded TV movies. It will be technically possible to capture TV movies and show them in the monitor.

Sound Volume Change. When a user strokes Rebo up and down, the volume indicator (a vertical bar) is shown on the video picture (Fig. 31). The length of the indicator is changed as the movement distance of the fingers is changed.

Turn On and Off. A series of screenshots in Fig. 4 shows a video picture being reduced in an image dimensions. When a user touches Rebo for more than one second, the image dimensions of the video picture are reduced and finally disappeared. If the user stops touching Rebo before the picture is disappeared, the video picture is enlarged and goes to an ordinary size.

On the other hand, a series of screenshots in Fig. [5 shows a video picture being enlarged. When the TV has been turned off, the picture is gradually enlarged while the user touches Rebo. When the user stops touching before the picture becomes to a maximum size, it is automatically going down and then disappeared.
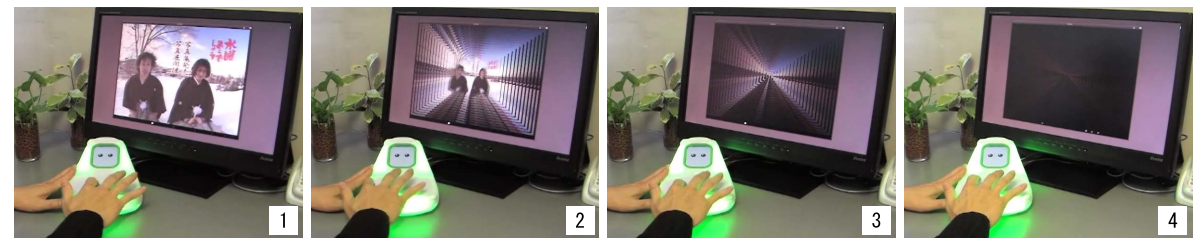

Fig. 5. Turn Off 


\section{Discussions}

Since Rebo utilizes a user's stroke with the fingers, he or she does not need to visually recognize and understand any icon, signal, and acknowledgment from a remote control. The user can concentrate on the feedback from appliances, but remote controls. The advantage of our approach partially resembles that of "eyes-free" approach 456] for many people including visually-impaired persons and elder people. Particularly for using mobile and wearable computers, input or output problems occur due to limited screen space and interaction techniques. They overcame the problems by developing a $3 \mathrm{D}$ audio radial pie menu and a sonically enhanced 2D gesture recognition system on a belt-mounted PDA. These previous studies attempted just to enrich usability of the user interface by touch-based devices like touch panels. However, we consider Rebo's touchbased approach is not only for usability of interface, but also for possibility for manual free appliances. When a user sees animations of the video picture such as sliding and zooming, she or he can understand the meaning of the feedback animation from the TV before the function is completely executed. Therefore, they can immediately notice the function of the appliance by stroking Rebo.

The feature of Rebo in comparison with a button-based remote control is the tolerance for mistakes. In our proposed system, a user concentrates on the feedback from a TV because he or she can operate Rebo with strokes without looking at it. The user can cancel the function before it is completely executed because he or she understands which function is executed by observing the feedback animation from the TV. For example, when the user wants to change the volume and operates Rebo, he or she tries other ways in stroking by observing the video picture moving left and right. However, in the button-based remote control, when the user pushes a button, the channel was changed immediately. The user has to search a button that tunes the previous channel and push it, and then search a button for changing the volume.

The tolerance for mistakes in Rebo enables unfamiliar users to home electric appliances to use it casually without a fear of making mistakes and unexpected behavior. We have a plan to investigate the effect of this tolerance in an experiment with elderly people.

\section{Conclusion}

In this paper, we propose a pet-like agent as a remote control for home electric appliances and develop a TV interaction system. Advantages of the proposed remote control are high familiarity and stroke operation. Life-like appearance and facial expression makes it familiar with users. We implemented facial expressions as a life-like behavior and video picture animations as feedback information from the TV to enable users to easily notice its functions. LED lighting provided Peripheral feedback for users to concentrate on the feedback from the appliance, but the remote control. The feature of Rebo in comparison with a button-based remote control is the tolerance for mistakes. This enables unfamiliar users to 
home electric appliances to use it casually without a fear of making mistakes and unexpected behavior.

In the next stage of our study, we have a plan to apply Rebo to various home electric appliances and enable users to seamlessly control them depending on the context.

\section{References}

1. LaPlant, B., Trewin, S., Zimmermann, G., Vanderheiden, G.: The universal remote console: A universal access bus for pervasive computing. In: IEEE Pervasive Computing, pp. 76-80 (2004)

2. MacIntyre, B., Mynatt, E.D., Voida, S., Hansen, K.M., Tullio, J., Corso, G.M.: Support for multitasking and background awareness using interactive peripheral displays. In: Proc. of the 14th annual ACM symposium on User interface software and technology, pp. 41-50 (2001)

3. Hsieh, G., Wood, K., Sellen, A.: Peripheral display of digital handwritten notes. In: Proc. of the SIGCHI conference on Human Factors in computing systems, pp. 285-288 (2006)

4. Zhao, S., Dragicevic, P., Chignell, M., Balakrishnan, R., Baudisch, P.: Earpod: eyesfree menu selection using touch input and reactive audio feedback. In: Proc. of the SIGCHI conference on Human factors in computing systems (CHI 2007), pp. 13951404 (2007)

5. Buil, V., Hollemans, G., van de Wijdeven, S.: Headphones with touch control. In: Proc. of the 7th international conference on Human computer interaction with mobile devices \& services, pp. 377-378 (2005)

6. Brewster, S., Lumsden, J., Bell, M., Hall, M., Tasker, S.: Multimodal 'eyes-free' interaction techniques for wearable devices. In: Proc. of the SIGCHI conference on Human factors in computing systems, pp. 473-480 (2003) 